\title{
CONFOR: A NEW PROGRAM FOR DETERMINING THE CONNECTION BETWEEN RADIO AND OPTICAL REFERENCE FRAMES
}

\author{
V.S. GUBANOV ${ }^{1}$ I.I. KUMKOVA ${ }^{1}$, AND V.V. TEL'NYUK-ADAMCHUK ${ }^{2}$ \\ 1 Institute of Applied Astronomy \\ 8 Zhdanovskaya ul. \\ 197042 Leningrad, USSR \\ 2 Astronomical Observatory of Kiev State University \\ Observatorna Str. 3 \\ 252035 Kiev, USSR
}

\begin{abstract}
The program for establishment of a link between the fundamental system FK5 and the radioastronomical coordinate system is described. The program includes photographic and meridian observations of extragalactic radio/optical sources and intermediate reference stars. Observatories of the USSR, GDR and Yugoslavia are participating in the project.
\end{abstract}

One of the possible ways to establish the link between the radio and optical coordinate systems is determining extragalactic radio source positions with reference to fundamental catalogue stars by means of photographic astrometry. This method has been used in 1980-1985 when the program ROAS (Radio/Optical Astrometric Sources) was fulfilled. The observational part of the program was based on the list of extragalactic radio sources recommended by IAU Commission 24 working group and included the determination of positions of 80 objects brighter than 18th stellar magnitude which are available for observations from the territory of the USSR. The observations were carried out using the Zeiss- 400 astrographs of the six observatories of the USSR. Final results of the program have shown the necessity of an expansion of the observations to more faint objects (fainter than $18^{\mathrm{m}}$ ) and also of providing a more reliable reference of intermediate stars to a fundamental catalogue.

Based on the results of ROAS [1] the new cooperative program was organized in 1988. It is called CONFOR - Connection of Frames in Optical and Radio regions. The program is supported by the Presidium of the USSR Academy of Sciences. This program is undertaken for the determination of relative orientation and errors of radioastronomical and optical coordinate systems and it consists of the following steps:

1. Determination of precise positions in the FK5 system of reference stars (up to $10^{\mathrm{m}}$ ) near radio sources;

2. Determination of positions of intermediate reference stars in the neighborhood of radio sources $\left(12^{\mathrm{m}}-14^{\mathrm{m}}, 16^{\mathrm{m}}-18^{\mathrm{m}}\right)$ in selected areas;

3. Determination of the extragalactic radio source positions with respect to the fundamental coordinate system FK5;

4. Designing and constructing a photoelectric camera [2] for observation of very faint objects against the background of bright catalogue stars. 
The observational part of the program CONFOR includes 200 central areas selected from the catalogue by Argue et al. [3]. The majority of objects on the list have brightness between 18 and 19 mag. The faintness of the objects is the main difficulty for obtaining their high-precision coordinates by means of photographic astrometry. That is why we use a two- or three-step method of reference. Constructing a new photoelectric camera which couples a CCD with a photographic plate will permit us to realize a direct connection between faint objects and bright catalogue stars.

Photographic observations of extragalactic radio sources are carried out by:

- 1-m R-C telescope of Sanglok Observatory, Astrophysical Institute of Academy of Sciences (AS) of Tajik SSR;

- 1-m R-C telescope of Astronomical Council AS of the USSR at Simeiz (since 1990);

- Schmidt telescope of Central Institute for Astrophysics AS of GDR;

- astrographs of Abastumani Observatory and at Maidanak;

- Shternberg State Astronomical Institute;

- 600-cm Zeiss telescope of the Main Astronomical Observatory of AS of the Ukrainian SSR.

The following telescopes are taking part in photographic observations of intermediate stars: astrographs of Astronomical Institute of AS of Uzbek SSR at Kitab, Astronomical Observatory of Kiev State University, of Abastumani observatory, of Shtemberg State Astronomical Institute.

The meridian observations are being provided by astronomical observatories of Kiev, Odessa and Kazan' universities and Belgrad Observatory.

As result of the completion of the program, the following will be received:

- catalogues of intermediate reference stars for stars brighter than $10^{\mathrm{m}}$ from meridian observations and for stars $12^{\mathrm{m}}-14^{\mathrm{m}}, 16^{\mathrm{m}}-18^{\mathrm{m}}$ from photographic observations;

- catalogue of extragalactic radiosources with respect to fundamental coordinate system FK5;

- the parameters of orientation and systematic errors of FK5-system reference to radioastronomical coordinate system;

- the model of the photoelectric camera and investigation of its astrometric possibilities.

\section{References}

1. Kumkova, I.I.: 1985, Izv. GAO AN SSSR Pulkovo, 203, 28 (in Russian).

2. Gubanov, V.S., Kumkova, I.I., Malachov, E.I. and Shornikov, O.E.: 1988, Preprint IPA AN SSSR, 2 (in Russian).

3. Argue, A.N., de Vegt, C., et al.: 1984, Astron. Astrophys. 130, 191.

\section{Discussion}

KopejKIN: How many reference objects have you in your program?

KumKova: About twelve intermediate reference objects. 